\title{
LINEAR FRACTIONAL TRANSFORMS \\ OF COMPANION MATRICES
}

\author{
by N. J. YOUNG
}

(Received 25 November, 1977)

Questions about polynomials can be turned into questions about matrices by associating with the polynomial

$$
p(z)=a_{0}+a_{1} z+\ldots+a_{n} z^{n} \quad\left(a_{n} \neq 0\right)
$$

(over an arbitrary field) its companion matrix

$$
T=\left[\begin{array}{ccccc}
0 & 1 & 0 & \cdots & 0 \\
0 & 0 & 1 & \cdots & 0 \\
\cdot & \cdot & \cdot & \cdots & \cdot \\
-a_{0} / a_{n} & -a_{1} / a_{n} & -a_{2} / a_{n} & \cdots & -a_{n-1} / a_{n}
\end{array}\right]
$$

which has $p / a_{n}$ as its characteristic polynomial. This technique is often used in stability theory, as indicated in [1]; companion matrices also occur in the theory of the rational canonical form.

In the study of root location for polynomials a common device is the use of the linear fractional transformation

$$
\phi(z)=(\alpha z+\beta) /(\gamma z+\delta) \quad(\alpha \delta-\beta \gamma \neq 0) .
$$

If one passes from $p$ to the polynomial

$$
q(z)=(-\gamma z+\alpha)^{n} p^{\circ} \phi^{-1}(z)
$$

one obtains a new polynomial $q$ whose roots are the images under $\phi$ of the roots of $p$. Thus the matrix

$$
\phi(T)=(\alpha T+\beta I)\left(\gamma T+\delta I^{-1},\right.
$$

provided it is defined, has the roots of $q$ as its eigenvalues, and with the right multiplicities. However, $\phi(T)$ is not in general the companion matrix of $q$, as virtually any example will show. How, then, can one obtain the companion matrix of $q$ from $\phi(T)$ ? It is a remarkable fact, proved by Shane and Barnett [1], that there is an $n \times n$ matrix $M_{\phi}$, depending only on $n$ and $\phi$, such that $M_{\phi} \phi(T) M_{\phi}^{-1}$ is a companion matrix for every companion matrix $T$. The proof in [1] proceeds by laborious computation of entries: indeed, the complexity is such that at one point the authors carry out the calculation for $n=3$ and leave the reader to convince himself that the procedure works in higher dimensions. The purpose of this note is to provide a proof which is simple and at the same time shows how to obtain $M_{\phi}$ more directly than by the method of [1].

Glasgow Math. J. 20 (1979) 129-132. 
THEOREM. Let $M_{\phi}$ be the $n \times n$ matrix $\left[m_{i j}\right]$, where, for $i=1,2, \ldots, n$,

$$
\sum_{i=1}^{n} m_{i j} z^{i-1}=(\gamma z+\delta)^{n-i}(\alpha z+\beta)^{i-1},
$$

$\phi$ being given by (3). Then $M_{\phi}$ is non-singular and, for any companion matrix $T$ such that $\gamma T+\delta I$ is non-singular, $M_{\phi} \phi(T) M_{\phi}^{-1}$ is again a companion matrix.

In fact (6) and (3) determine $M_{\phi}$ only up to a scalar multiple, but this is clearly unimportant.

We shall use tensor product notation: if $P, Q$ are $n \times n$ matrices then $P \otimes Q$ denotes the $n^{2} \times n^{2}$ matrix which, written in block form, has $p_{i j} Q$ as the $n \times n$ block in the $(i, j)$ position, where $P=\left[p_{i j}\right]$. It is also convenient to introduce the notation

so that $H(P)$ is of type $n^{2} \times n$.

$$
H(P)=\left[\begin{array}{c}
I \\
P \\
P^{2} \\
\vdots \\
P^{n-1}
\end{array}\right],
$$

The proof of the theorem is based on the following characterization of companion matrices.

LEMMA. Let $B$ be an $n \times n$ matrix whose minimal polynomial $p$ is of degree $n$. An $n \times n$ matrix $X$ is the companion matrix of $p$ if and only if

$$
H(B) B=(X \otimes I) H(B) \text {. }
$$

The proof is immediate on writing down both sides of (8) in block form.

Proof of Theorem. Let $T$ be a companion matrix such that $\phi(T)$ is defined, and write $B=\phi(T)$. One can see by considering the first row that the matrices $I, T, \ldots, T^{n-1}$ are linearly independent, and since $A$ and $\phi(A)$ have minimal polynomials of the same degree, for any $A$, it follows that $B$ also has minimal polynomial of degree $n$.

Equation (8) implies in particular that

and hence

$$
H(T) T=(T \otimes I) H(T)
$$

$$
H(T)(\alpha T+\beta I)=((\alpha T+\beta I) \otimes I) H(T) .
$$

Replace $\alpha, \beta$ by $\gamma, \delta$ respectively, premultiply by $(\gamma T+\delta I)^{-1} \otimes I$ and postmultiply by $(\gamma T+\delta I)^{-1}$ to obtain

$$
H(T)(\gamma T+\delta)^{-1}=\left((\gamma T+\delta I)^{-1} \otimes I\right) H(T) .
$$

Combining this with (9), we have

$$
H(T) B=(B \otimes I) H(T) .
$$


From the definition of $M_{\phi}$ one has

$$
\begin{aligned}
H(B)(\gamma T+\delta I)^{n-1} & =\left[\begin{array}{c}
(\gamma T+\delta I)^{n-1} \\
(\gamma T+\delta I)^{n-2}(\alpha T+\beta I) \\
\cdots \\
(\alpha T+\beta I)^{n-1}
\end{array}\right] \\
& =\left(M_{\phi} \otimes I\right) H(T)
\end{aligned}
$$

Since $B$ commutes with $(\gamma T+\delta I)$, we have

$$
H(B) B=\left(M_{\phi} \otimes I\right) H(T) B(\gamma T+\delta I)^{-n+1},
$$

and now (10) yields

$$
\begin{aligned}
H(B) B & =\left(M_{\phi} \otimes I\right)(B \otimes I) H(T)(\gamma T+\delta I)^{-n+1} \\
& =\left(M_{\phi} B \otimes I\right) H(T)(\gamma T+\delta I)^{-n+1} .
\end{aligned}
$$

Suppose for the moment that $M_{\phi}$ is non-singular: then (11) tells us that

$$
H(T)(\gamma T+\delta I)^{-n+1}=\left(M_{\phi}^{-1} \otimes I\right) H(B),
$$

and, combining this with (12), we find that

$$
H(B) B=\left(M_{\phi} B M_{\phi}^{-1} \otimes I\right) H(B) .
$$

An application of the lemma now shows that $M_{\phi} B M_{\phi}^{-1}$ is a companion matrix, as was claimed.

It remains to show that $M_{\phi}$ is non-singular. This is most neatly accomplished by proving that $\phi \rightarrow M_{\phi}$ is a representation of GL(2). To see this write the definition (6) of $M_{\phi}$ in the form

$$
M_{\phi}\left[\begin{array}{c}
1 \\
z \\
\vdots \\
z^{n-1}
\end{array}\right]=(\gamma z+\delta)^{n-1}\left[\begin{array}{c}
1 \\
\phi(z) \\
\vdots \\
\phi(z)^{n-1}
\end{array}\right]
$$

Then, if $\phi^{\prime}(z)=\left(\alpha^{\prime} z+\beta^{\prime}\right)\left(\gamma^{\prime} z+\delta^{\prime}\right)^{-1}$, we have

$$
\begin{gathered}
M_{\phi^{\prime}} M_{\phi}\left[\begin{array}{c}
1 \\
z \\
\vdots \\
z^{n-1}
\end{array}\right]=(\gamma z+\delta)^{n-1}\left(\gamma^{\prime} \phi(z)+\delta^{\prime}\right)^{n-1}\left[\begin{array}{c}
1 \\
\phi^{\prime} \circ \phi(z) \\
\vdots \\
\phi^{\prime} \circ \phi(z)^{n-1}
\end{array}\right] \\
=\left\{\left(\gamma^{\prime} \alpha+\delta^{\prime} \gamma\right) z+\gamma^{\prime} \beta+\delta^{\prime} \delta\right\}^{n-1}\left[\begin{array}{c}
1 \\
\phi^{\prime} \circ \phi(z) \\
\vdots \\
\phi^{\prime} \circ \phi(z)^{n-1}
\end{array}\right]=M_{\phi^{\prime} \circ \phi}\left[\begin{array}{c}
1 \\
z \\
\vdots \\
z^{n-1}
\end{array}\right] .
\end{gathered}
$$


Thus $M_{\phi}, M_{\phi}=M_{\phi^{\prime} \phi}$, and it is easy to see that if $\phi(z)=z$, then $M_{\phi}=I$. It follows that $M_{\phi}$ is non-singular, and $M_{\phi}^{-1}=M_{\phi^{-1}}$ (to make this precise we should normalize by requiring $\alpha \delta-\beta \gamma=1$ ). This completes the proof of the theorem, and also shows how to obtain $M_{\phi}^{-1}$ easily: the entries in the $i$ th row of $M_{\phi}^{-1}$ are the coefficients in the polynomial

$$
(\alpha \delta-\beta \gamma)^{-n+1}(-\gamma z+\alpha)^{n-i}(\delta z-\beta)^{i-1} .
$$

We can re-state our conclusions in the following way. Write $\tilde{\phi}(X)=M_{\phi} \phi(X) M_{\phi}^{-1}$, whenever $\phi(X)$ is defined. The mapping $\phi \rightarrow \tilde{\phi}$ is an isomorphism of GL(2) onto a group of (non-linear) transformations of the space of $n \times n$ matrices which leaves invariant the set of companion matrices. When the field in question is $\mathbf{C}$ the elements of the image group are bianalytic transformations.

Notice a special case: if $\phi(z)=1 / z$, then it is easy to see that $M_{\phi}$ is the matrix with ones on the principal cross-diagonal and zeros everywhere else. It follows that if $T$ is the companion matrix of a polynomial $p$ for which $p(0) \neq 0$ then $T^{-1}$ is the matrix obtained by reversing the order of both rows and columns in the companion matrix of the polynomial $z^{n} p(1 / z)$. This fact can easily be checked directly.

\section{REFERENCE}

1. B. A. Shane and S. Barnett, On the bilinear transformation of companion matrices, Linear Algebra and Appl. 9 (1974), 175-184.

UNIVERSITY OF GLASGOW

UNIVERSITY GARDENS

GLASGOW

SCOTLAND

G12 8QW 